\title{
Dental and Oral Health Practices for Sound Gums
}

\section{Arpita Upadhyay* and Amitha Ramesh}

Periodontics, Villoo Poonawalla Memorial Hospital, Pune, India

*Corresponding Author: Arpita Upadhyay, Periodontics, Villoo Poonawalla Memorial Hospital, Pune, India.

Received: October 14, 2019; Published: November 05, 2019

DOI: $10.31080 /$ ASDS.2019.03.0693

\begin{abstract}
Gingival and periodontal disease has affected humans since the dawn of civilisation. Tooth loss and loss of function are the common sequelae of periodontal disease. The primary reason for maintaining oral health and undergoing regular oral prophylaxis by a professional is reduction and elimination of etiological and contributing factors in periodontal treatment.

This review article deals with the various methods of mechanical plaque control as well as the latest advances in the field.

Keywords: Mechanical Plaque Control; Toothbrush; Flossing Devices; Oral Irrigation Devices
\end{abstract}

\section{Introduction}

Gingival and periodontal disease, in their various forms have afflicted humans since the dawn of history. Studies in paleopathology have indicated that destructive periodontal disease, as evidenced by bone loss, affected humans in such diverse cultures as ancient Egypt and Pre-Columbian America. The earliest historical records dealing with medical topics reflect an awareness of periodontal disease and need for treatment. However, methodical, carefully reasoned, therapeutic discussions did not exist until the Arabic treatise of the middle ages, and modern treatment, with illustrated text and sophisticated instrumentation, did not develop until the time of Pierre Fauchard [1] in the eighteenth century.

Oral Hygiene was practised by the early Sumerians of 3000 B.C., and elaborately decorated gold toothpicks found in excavations at Mesopotamia suggest an interest in cleanliness of the mouth. The Babylonians and Assyrians like the early Sumerians, suffered from periodontal problems, and a clay tablet of the period tells of treatment by gingival massage combined with various herbal medication $[2,3]$.

Similar mentions about gingival inflammation, Periodontal disease and tooth loss have been found in Indian and Chinese inscriptions, including descriptions of loose teeth and purulent discharge from the gingiva, stressing toothbrushing and oral hygiene.

\section{Rationale for oral prophylaxis}

According to American Dental Association (ADA), "oral health is a functional, structural, aesthetic, physiologic, and psychosocial state of well-being and is essential to an individual's general health and quality of life. The primary reason for maintaining oral health and undergoing regular oral prophylaxis by a professional is reduction and elimination of etiological and contributing factors in periodontal treatment. This is achieved by complete removal of calculus and institution of a comprehensive daily plaque control regimen $[4,5]$.

Brushing twice daily along with use of floss and mouth wash on a regular basis are important aspects of maintaining oral hygiene and it remains of utmost importance for every clinician to motivate and educate the patient to follow through for long term maintenance. However, it requires commitment by the patient to change daily habits and return for regular visits for maintenance and reinforcement. 


\section{How to raise awareness}

Various studies conducted have proved that the oral health awareness and practices among the general population was poor and needed to improve. About $69.20 \%$ of the participants used a toothbrush with toothpaste as a method of cleaning their teeth; $35.71 \%$ brushed twice in a day; $33.03 \%$ brushed both in the morning and at bedtime; and 8.93\% used mouthwash. About $40.62 \%$ visited the dentist during the last six months; among them $61.18 \%$ attended because of pain. Almost three-fourth of the participants knew that tooth decay and bad breath were the effects of not cleaning the teeth. It was known to 71.42, 63.39, 70.53, and $73.21 \%$ of the respondents, respectively, that excess sweet, cold drink, alcohol, and smoking/pan chewing were bad for dental health. Television was the source of knowledge to $57.14 \%$ of the participants and $35.71 \%$ acquired their knowledge from a dentist. Females, literates, urban residents, users of mouthwash, and regular visitors to the dentist had good oral hygiene practices [6].

Health education and preventive dental care interventions not only reduce the incidence of oral diseases but are also costeffective, easy to administer, and logical to use at community level [7]. The use of study models to demonstrate proper tooth brushing techniques on patient visits goes a long way in oral hygiene maintenance. In the rapidly advancing scientific era, educational videos have emerged as relatively popular tool for imparting health education. The embedding of video in bringing oral health awareness can make a significant difference in the knowledge and attitude of the public. Video enhances, dramatizes, and brings a sense of realism, which can have a great impact on the population. Hence, videotape can be a useful adjunct in teaching about oral health in Indian setup/population [8].

\section{Methods of mechanical plaque control}

We already know about the common mechanical plaque control aids that function with the aim of day to day oral hygiene maintenance. These include:

- $\quad$ Chewing Sticks

- Toothbrushes

$\circ \quad$ Depending on the type of bristle- Natural and Synthetic

○ Depending of the Function- Manual and Powered

○ Depending on the bristle type- Soft, Medium and Hard
○ Depending on the number of tufts- Single and Multi Tufted

- Interdental Aids

○ Dental Floss/Tape

- $\quad$ Twisted, Non Twisted

- Waxed, Unwaxed

- Bonded, Non Bonded

- Thick, Thin

- Monofilament floss

- Manual Floss

- $\quad$ Powered Floss

○ Interdental Brushes

- Cone

- Cylindrical

- Small head with reversible handle

- Brushes with wire handle

- Single tufted marginal

- Multitufted Interdental

- $\quad$ Toothpicks

- Wooden tips

- $\quad$ Rubber tips

The modern toothbrush- Heralding a new Era of plaque removal!

Since the time of invention till the present day, toothbrushes have been continuously improved to promote healthier teeth. The modern, manual toothbrush helps to clean teeth more effectively than traditional instruments such as fingers, sticks, and twigs. As a result of continuous research and development, recently more advanced toothbrushes such as electric rotation oscillation, sonic, and solar powered toothbrushes have been invented. These advanced toothbrushes do not just restrict their functions of scrubbing teeth with a plastic handle; they also promote better oral healthy with less effort using their additional cleaning mechanisms.

\section{Powered toothbrushes}

The powered toothbrushes are provided with a brush head capable of a variety of motions driven by a power source. Powered brushes were first introduced with a back and forth action. Subsequent development has led to the evolution of rotary action brushes, and more recently higher frequency vibration brushes. 
The head of the toothbrush is more compact then the manual, conventional toothbrush and has more compact, single tufts for easier access to interproximal as well as the more inaccessible distal areas of the oral cavity.

It has been observed that there is not much clinically significant improvement in plaque control and gingival health when using a powered toothbrush over the conventional, manual toothbrush $[9,10]$. However, such toothbrushes have been found to be effective in persons not highly motivated to oral health care, including children and Geriatric patients. In addition, such toothbrushes can be effective in patients with limited manual dexterity.

\section{Ultrasonic toothbrush}

The ultrasonic toothbrush is a manual toothbrush, in which a Piezoelectric ultrasonic emitter is embedded in the brush head. The ultrasonic emitter is driven by a power supply located in the handle that operates at $1.6 \mathrm{MHz}$.

Studies have shown that the effect of such toothbrushes on the oral hygiene status and stain removing efficacy is better in comparison to manual toothbrushes. It is also beneficial in destroying bacteria, removal of stains of coffee, wine, nicotine, and food. The teeth returned to their natural white colour within days. It also worked under braces, cleaning, and removing stains. It is gentle for children. It also cleans gingival pockets where even dental floss cannot reach. It is gentle enough to use immediately after oral surgery, including implants, avoiding damage to teeth, and gums and is painless on sensitive teeth and gums [11-13].

\section{Ionic toothbrush}

Ionic toothbrush works on the principle of polarity that every element in nature has a positive or a negative charge. The ionic toothbrush is only slightly larger than the manual toothbrush, with replaceable brush heads. It works on the principle of changing surface charge of tooth to repel plaque even from inaccessible areas of teeth. The bonding between the pellicles and bacteria is mediated by $\mathrm{Ca} 2+$ bridge formation. The anions supplied by the lithium battery inhibits the bonding between the bacteria and $\mathrm{Ca} 2+$ and prevents the bacteria from adsorbing to the pellicles. Hence, the plaque accumulation is reduced because the above mentioned anions continuously supplied from the tips of the bristles of the ionic toothbrushes prevent the mild electrostatic bonding between the bacteria itself.
It was observed that there was a significant reduction in plaque index and gingival index scores as well as there was no soft tissue trauma following the use of ionic toothbrushes.

\section{Laser toothbrushes}

Dentinal hypersensitivity is one of the most common complications that affect patients both in day to day life as well as after periodontal therapy. It has been shown that Nd: YAG lasers can block depolarization of rapidly conducting A- $\beta$ fibers, and such laser radiation can block an action potential conduction in a simulated interdental nerve.

Laser toothbrushes are an improved version of the modern toothbrush that emits red (635 $\mathrm{nm}$ ) light in the visible spectrum produced by a diode laser inside the toothbrush powered with an AA battery. The LLLT with the help of such toothbrushes help to reduce dentinal hypersensitivity $[14,15]$. Another advantage of using laser in toothbrush is that the patient can use it at home, which is cost effective, less time consuming and easily used by patients.

\section{Powered flossing devices}

Dental floss is the mostly widely recommended tool for removing plaque from proximal surfaces. Dental floss as an adjunct to tooth brushing has a significant effect in the reduction of plaque and parameters of gingival inflammation, in adults with periodontal disease. However, at the same time, manual flossing is a very technique sensitive procedure that requires patient knowledge and awareness. To overcome such issues, powered flossing has been introduced. They consist of battery operated nylon tip that slip easily between teeth and is very gentle to the gums. They are very effective in cases of patients wearing orthodontic appliances. It was observed that powered flossing devices were just as effective as manual floss techniques for removal of plaque but were preferred as lesser manual dexterity and technique sensitivity was needed in this case [16].

\section{Oral irrigation devices}

A dental water jet or water flosser or oral irrigator (OI) is an electric device that delivers a pulsating stream of fluid (containing chlorhexidine, stannous fluoride, iodine solution, antibiotics like $5 \%$ tetracycline hydrochloride) through controlled pressure which is aimed at the removal of interdental and subgingival plaque biofilm on tooth surfaces to reduce inflammation as an adjunct to 
tooth brushing. The devices may be power or non-power driven and depending on the type of application; they are designed for both professional as well as patient applied home irrigation.

The two main physical features of water irrigation action include pulsation and pressure. Pressure is essentially regulated by pulsation. A combination of these two actions causes disruption of bacterial activity, expulsion of subgingival bacteria, and the removal of loosely lodged debris and food particles. Clinical effectiveness has been demonstrated in the 50 - 90 psi (pounds of pressure per square inch) range [17].

\section{Rubber tip stimulators}

The rubber tip stimulator is an excellent tool for removing plaque from around the gum line and also for stimulating blood flow to the gums. The rubber tip stimulator should be traced gently along the outer and inner gum line at least once each day. Any plaque on the tip can be rinsed off with tap water. It is important to replace the tip as soon as it starts to appear worn, and to store the stimulator in a cool, dry place.

\section{Tongue cleaners}

Tongue cleaners are special devices which have been designed to remove the build-up of bacteria, fungi and food debris from the tongue surface. The fungi and bacteria that colonize on the tongue have been related to halitosis (bad breath) and a great many systemic diseases like diabetes, heart disease, respiratory disease and stroke. Tongue cleaners can be made from metal, wood or plastic and shaped in accordance with the contours of the tongue. Tongue cleaning should be done prior to brushing to prevent the ingestion of fungi and bacteria.

\section{Conclusion}

Mechanical Plaque Removal is the primary objective of oral hygiene practices. With each day, newer and more advanced methods are being formulated with the main aim of reducing the effort and time needed for oral hygiene maintenance. Patient education and raising awareness should be an important objective for every dental surgeon to fight the good fight and make the world a better place one oral cavity at a time.

\section{Bibliography}

1. Fauchard P "Le Chirurgein Dentiste, ou Traite des Dents, Paris, J Maruiette (Reprinted in facsimile, Paris, Prelat,1961; English translation by L Lindsay, London, 1946, Butterworth and Co (1928).
2. Guerini V. "History of Dentistry". Philadelphia, Lea and Febinger (1909).

3. Jastrow N. "The medicine of Babylonians and Assyrians". Proceedings of the Royal Society of Medicine 7 (1914): 109-176.

4. Pihlstrom BL and Ammons WF. "Position Paper: Treatment of Gingivitis and Periodontitis". Journal of Periodontology 68.12 (1997):1246-1253.

5. Anderson JL. "Integration of Plaque Control into Practice of Dentistry". Dental Clinics of North America 16.4 (1972): 621630.

6. Paul B., et al. "Awareness and Practices of Oral Hygiene and its Relation to Sociodemographic Factors among Patients attending the General Outpatient Department in a Tertiary Care Hospital of Kolkata, India". Journal of Family Medicine and Primary Care 3.2 (2014): 107-111.

7. Hebbal M., et al. "Evaluation of knowledge and plaque scores in school children before and after health education". Dental Research Journal (Isfahan) 8.4 (2011): 189-196.

8. Naseem Shah., et al. "Effectiveness of an educational video in improving oral health knowledge in a hospital setting". Indian Journal of Dental 7.2 (2016): 70-75.

9. Silverman J., et al. "Comparison of powered and manual toothbrushes for plaque removal by 4 to 5 year old children". Pediatric Dentistry Journal 26 (2004): 225-230.

10. Vibhute A and Vandana KL. "The effectiveness of manual versus powered toothbrushes for plaque removal and gingival health: A meta analysis". Journal of Indian Society of Periodontology 16 (2012): 156-160.

11. Vandana KL and Penumatsa GS. "A comparative evaluation of an ultrasonic and a manual toothbrush on the oral hygiene status and stain removing efficacy". Journal of Indian Society of Pedodontics and Preventive Dentistry 22 (2004): 33-35.

12. Sharma K and Sangwan A. "Era of smart toothbrushes". Advances in Human Biology 3 (2013): 2.

13. Deshmukh J., et al. "Clinical evaluation of an ionic tooth brush on oral hygiene status, gingival status, and microbial parameter". Indian Journal of Dental Research 17 (2006): 74-77. 
14. Ko Y., et al. "Treatment of dentin hypersensitivity with a low level laser emitting toothbrush: Double blind randomised clinical trial of efficacy and safety". Journal of Oral Rehabilitation 4 (2014): 523-531.

15. Yaghini J., et al. "Evaluation of the effect of low level laser therapy toothbrush in treatment of dentin hypersensitivity". Journal of Lasers in Medical Sciences 6 (2015): 85-91.

16. Shibly 0., et al. "Clinical evaluation of an automatic flossing device vs. Manual flossing". The Journal of Clinical Dentistry 12 (2001): 63-66.

17. Bhaskar SN., et al. "Water jet devices in dental practice". Journal of Periodontology 42 (1971): 658-664.

Volume 3 Issue 12 December 2019

(c) All rights are reserved by Arpita Upadhyay and

Amitha Ramesh.

Citation: Arpita Upadhyay and Amitha Ramesh. "Dental and Oral Health Practices for Sound Gums”. Acta Scientific Dental Sciences 3.12 (2019): $23-27$. 